\title{
Status and prospects of CDEX: the China Dark Matter Experiment
}

\author{
Li, Hau-Bin*† \\ Institute of Physics, Academia Sinica, Taipei \\ E-mail: lihb@gate.sinica.edu.tw
}

\begin{abstract}
Germanium detectors with sub-keV sensitivities [1,2,3] offer a unique opportunity to search for light WIMP Dark Matter and axion-like particles. We will highlight our results and status of CDEX dark matter experiment $[4,5,6,7,8,9]$ at the China Jinping Underground Laboratory (CJPL) [10] in China. The detector R\&D programs which allow us to experimentally probe this new energy window will be discussed, especially the new bulk/surface events separation scheme. Recent results from axion searches, as well as results from dark matter searches [4, 5, 6, 7, 8, 9] will be described. Status of the construction of CJPL-II will be presented.
\end{abstract}

ICHEP 2017, XXXIX International Conference on High Energy Physics

4-11 July 2018

Seoul, Republic of Korea

\footnotetext{
* Speaker.

${ }^{\dagger}$ on bahalf Qian Yue, Tsinghua U. \& CDEX collaboration.
} 


\section{Introduction}

China Dark matter EXperiment (CDEX) ultilize point-contact Germanium detector ( $p \mathrm{Ge})$ to search for WIMP in China Jinping Underground Laboratory (CJPL). Cosmic ray rate at CLPJ is $<0.2$ muons $/ \mathrm{m}^{2} /$ day at depth of $6720 \mathrm{~m}$. w. e., making it the ideal location for WIMP searches and neutrinoless double-beta decay experiments.

For WIMP searches, $p \mathrm{Ge}$ is particular sensitive to WIMP mass at few to few tens $\mathrm{GeV}$, ideal to probe the regions with positive dark matter signals suggested by DAMA/LIBRA and CoGeNT experiments.

A particular important feature of $p \mathrm{Ge}$ is bulk/surface events separation $[1,2,3]$, which contribute to the largest uncertainties in analysis.

\section{CDEX experiments}

\subsection{CDEX-1A \& CDEX-1B experiments}

The CDEX-1A and CDEX-1B experiments are two phases of the $\sim 1 \mathrm{~kg}$ targer mass CDEX experiment, with detection threshold of $450 \mathrm{eV}$ and $160 \mathrm{eV}$, respectively. Both detector targets are $\sim 1 \mathrm{~kg}$ of mass with $\sim 1 \mathrm{~mm}$ of dead layers, shielded with $20 \mathrm{~cm}$ of copper, $20 \mathrm{~cm}$ of borated polyethylene and $20 \mathrm{~cm}$ of lead, from inside to outside. Both experiments are ran side-by-side inside a $6 \mathrm{~m}(\mathrm{H}) \times 8 \mathrm{~m}(\mathrm{~L}) \times 4 \mathrm{~m}(\mathrm{~W})$ polyethylene room with wall thickness of $1 \mathrm{~m}[4,6,8]$.

Results on unmodulated spin-independent (SI) and spin-dependent (SD) cross-sections by the $\chi \mathrm{N}$ recoil spectral analysis were published in [4, 6] (CDEX-1A) and [8] (CDEX-1B), in particular both DAMA/LIBRA and CoGeNT positive claims are well excluded. Results of axion searches on CDEX-1A data were published in [7].

The data taking in CDEX-1B experiment is ongoing, 3 years live-time of data (till October of 2018) had been accumulated, annual modulation analysis is underway.

\subsection{CDEX-10 experiment}

Toward future ton-scale DM experiment, the second generation of CDEX experiment with a total detector mass of about $10 \mathrm{~kg}$, called CDEX-10, has used three triple-element pPCGe strings directly immersed in liquid nitrogen (LN2), denoted as C10A (B,C), as shown in Fig. 1. Compared with cold finger cooling and high- $Z$ material shielding systems, low-Z material shielding, such as with LN2 or liquid argon, provides better control of radiation background.

CDEX-10 focuses on the arraying technologies and background understanding of the prototype pPCGe detectors developed based on the CDEX-1 technique. The new CDEX-10 array detectors and dedicated data acquisition (DAQ) system started testing and data-taking inside a LN2 tank in 2016 at CJPL. C10A was returned to the CANBERRA factory in France for upgrades. Unmodulated spin-independent (SI) (Fig. 2) and spin-dependent (SD) cross-sections by the $\chi \mathrm{N}$ recoil results on one of the crystal (C10B-Ge1 detector) were published in [9].

\section{Prospect}

The CDEX experiment continues taking data at CJPL, expanding to Ge-detector arrays immersed in liquid nitrogen both as cryogenic coolant and shielding [9]. R\&D efforts on acquisition 


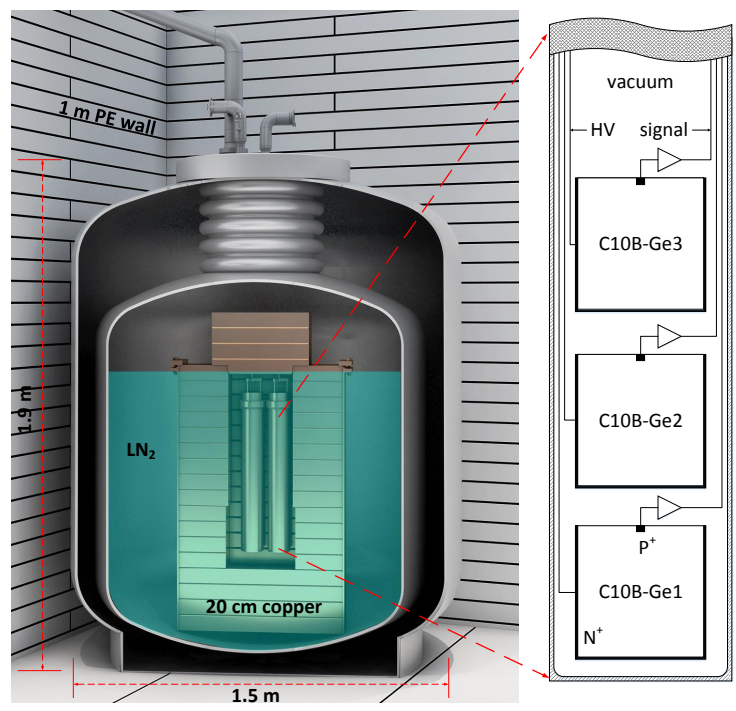

Figure 1: Configuration of CDEX-10 experimental setup (left) and C10B detector layout inside the string (right). $\mathrm{C} 10 \mathrm{~B}$ and $\mathrm{C} 10 \mathrm{C}$ are running inside the $\mathrm{LN} 2$ tank which has an outer diameter of $1.5 \mathrm{~m}$ and a height of $1.9 \mathrm{~m}$. Each detector string consists of three $p \mathrm{Ge}$ detectors tagged as Ge1 to Ge3 from bottom to top. The size of each germanium crystal is approximately $62 \mathrm{~mm} \times 62 \mathrm{~mm}$.

of the Ge-detector fabrication technology, as well as further reduction of ambient and internal background, are being pursued. Scaled-up experiment towards target mass of $100 \mathrm{~kg}$ are being prepared at CJPL-Phase 2 [10].

\section{References}

[1] H. B. Li, et. al., Differentiation of Bulk and Surface Events in p-type Point-Contact Germanium Detectors for Light WIMP Searches, Astropart. Phys. 56 (2014) 1

[2] A. K. Soma, et. al., Characterization and Performance of Germanium Detectors with sub-keV Sensitivities for Neutrino and Dark Matter Experiments, Nucl. Instrum. Meth. A 836 (2016) 67

[3] L. T. Yang, et. al., Bulk and Surface Event Identification in p-type Germanium Detectors, Nucl. Instrum. Meth. A $\mathbf{8 8 6}$ (2018) 13

[4] Q. Yue, et. al., Limits on light weakly interacting massive particles from the CDEX-1 experiment with a p-type point-contact germanium detector at the China Jinping Underground Laboratory, Phys. Rev. D 90 (2014) 091701(R)

[5] S. K. Liu, et. al., Limits on light WIMPs with a germanium detector at 177 eVee threshold at the China Jinping Underground Laboratory, Phys. Rev. D 90 (2014) 032003

[6] W. Zhao, et. al., Search of low-mass WIMPs with a p-type point contact germanium detector in the CDEX-1 experiment, Phys. Rev. D 93 (2016) 092003

[7] S. K. Liu, et. al., Constraints on axion couplings from the CDEX-1 experiment at the China Jinping Underground Laboratory, Phys. Rev. D 95 (2017) 052006

[8] L. T. Yang, et. al., Limits on light WIMPs with a $1 \mathrm{~kg}$-scale germanium detector at 160 eVee physics threshold at the China Jinping Underground Laboratory, Chin. Phys. C 42 (2018) 23002 
(a)

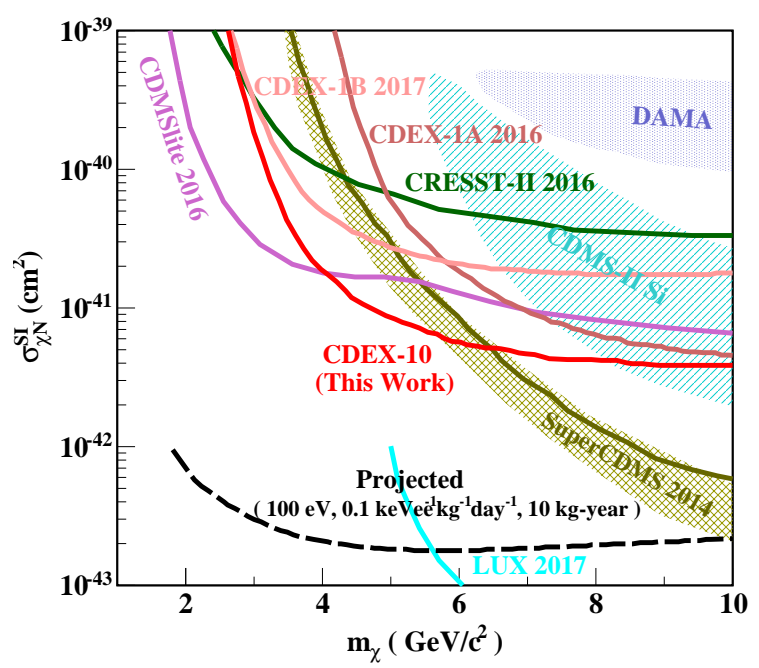

Figure 2: Exclusion plots of SI $\chi$-N coupling at $90 \%$ confidence level, superimposed with results from other benchmark experiments ([9] for detail explanation).

[9] H. Jiang, et. al., Limits on Light Weakly Interacting Massive Particles from the First $102.8 \mathrm{~kg} \times$ day Data of the CDEX-10 Experiment, Phys. Rev. Lett. 120 (2018) 241301

[10] C. P. Cheng, et. al., The China Jinping Underground Laboratory and Its Early Science, Ann. Rev. Nucl. Part. Sci. 67 (2017) 231 